\title{
Smiling for the Delayed Volatility Swaps
}

\author{
Anatoliy Swishchuk ${ }^{1}$ and Nelson Vadori ${ }^{2}$ \\ 1 Corresponding author. University of Calgary, Department of Mathematics, 2500 \\ University Drive NW, Calgary, AB, Canada T2N 1N4;e-mail: aswish@ucalgary.ca; \\ Tel.: 1(403)220-3274; Fax: 1(403) 282-5150. \\ 2 University of Calgary, Department of Mathematics, e-mail: nvadori@ucalgary.ca.
}

\begin{abstract}
We present a variance drift adjusted version of the Heston model which leads to a significant improvement of the market volatility surface fitting (compared to Heston). The numerical example we performed with recent market data shows a significant reduction of the average absolute calibration error ${ }^{1}$ (calibration on 12 dates ranging from Sep. $19^{\text {th }}$ to Oct. $17^{\text {th }} 2011$ for the FOREX underlying EURUSD). Our model has two additional parameters compared to the Heston model, can be implemented very easily and was initially introduced for volatility derivatives pricing purpose. The main idea behind our model is to take into account some past history of the variance process in its (risk-neutral) diffusion. Using a change of time method for continuous local martingales, we derive a closed formula for the Brockhaus\&Long approximation of the volatility swap price in this model. We also consider dynamic hedging of volatility swaps using a portfolio of variance swaps.
\end{abstract}

Keywords: variance swap; volatility swap; stochastic volatility with delay; Heston model; change of time; dynamic hedging.

\section{Introduction}

The volatility process is an important concept in financial modeling as it quantifies at each time $t$ how likely the modeled asset log-return is to vary significantly over some short immediate time period $[t, t+\epsilon]$. This process can be stochastic or deterministic, e.g. local volatility models in which the (deterministic) volatility depends on time and spot price level. In quantitative finance, we often consider the volatility process $\sqrt{V_{t}}$ (where $V_{t}$ is the variance process) to be stochastic as it allows to fit the observed vanilla option market prices with an acceptable bias as well as to model the risk linked with the future evolution of the volatility smile (which deterministic model cannot), namely the forward smile. Many derivatives are known to be very sensitive to the forward smile, one of the most popular example being the cliquet options (options on future asset performance, see Kruse and Nögel [18] for example).

Heston model (Heston [11]; Heston and Nandi [12]) is one of the most popular stochastic volatility models in the industry as semi-closed formulas for vanilla option prices are available, few (five) parameters need to be calibrated, and it

1 The average absolute calibration error is defined to be the average of the absolute values of the differences between market and model implied Black \& Scholes volatilities. 
accounts for the mean-reverting feature of the volatility.

One might be willing, in the variance diffusion, to take into account not only its current state but also its past history over some interval $[t-\tau, t]$, where $\tau>0$ is a constant and is called the delay. Starting from the discrete-time GARCH(1,1) model (Bollerslev [4]), a first attempt was made in this direction in Kazmerchuk et al. [16], where a non-Markov delayed continuous-time GARCH model was proposed $\left(S_{t}\right.$ being the asset price at time $t$, and $\gamma, \theta, \alpha$ some positive constants):

$$
\frac{d V_{t}}{d t}=\gamma \theta^{2}+\frac{\alpha}{\tau} \ln ^{2}\left(\frac{S_{t}}{S_{t-\tau}}\right)-(\alpha+\gamma) V_{t}
$$

this model being inherited from its discrete-time analogue (where $L$ is a positive integer):

$$
\sigma_{n}^{2}=\tilde{\gamma} \tilde{\theta}^{2}+\frac{\tilde{\alpha}}{L} \ln ^{2}\left(\frac{S_{n-1}}{S_{n-1-L}}\right)+(1-\tilde{\alpha}-\tilde{\gamma}) \sigma_{n-1}^{2} .
$$

The parameter $\theta^{2}$ (resp. $\gamma$ ) can be interpreted as the value of the long-range variance (resp. variance mean-reversion speed) when the delay is equal to 0 (we will see that introducing delay modifies the value of these two model features), and $\alpha$ a continuous-time equivalent of the variance $\operatorname{ARCH}(1,1)$ autoregressive coefficient. In fact, we can interpret the right-hand side of previous diffusion equation as the sum of two terms:

- the delay-free term $\gamma\left(\theta^{2}-V_{t}\right)$ which accounts for the mean-reverting feature of the variance process

- $\alpha\left(\frac{1}{\tau} \ln ^{2}\left(\frac{S_{t}}{S_{t-\tau}}\right)-V_{t}\right)$ which is a pure (noisy) delay term, i.e. that vanishes when $\tau \rightarrow 0$ and takes into account the past history of the variance (via the term $\left.\ln \left(\frac{S_{t}}{S_{t-\tau}}\right)\right)$. The autoregressive coefficient $\alpha$ can be seen as the amplitude of this pure delay term.

In Swishchuk [23] and Swishchuk and Li [21], the authors point out the importance to incorporate the real world $\mathbb{P}-\operatorname{drift} d_{\mathbb{P}}(t, \tau):=\int_{t-\tau}^{t}\left(\mu-\frac{1}{2} V_{u}\right) d u$ of $\ln \left(\frac{S_{t}}{S_{t-\tau}}\right)$ in the model (where $\mu$ stands for the real world $\mathbb{P}$-drift of the stock price $S_{t}$ ), transforming the variance dynamics into:

$$
\frac{d V_{t}}{d t}=\gamma \theta^{2}+\frac{\alpha}{\tau}\left[\ln \left(\frac{S_{t}}{S_{t-\tau}}\right)-d_{\mathbb{P}}(t, \tau)\right]^{2}-(\alpha+\gamma) V_{t}
$$

The latter diffusion (3) was introduced in Swishchuk [23] and Kazmerchuk et al. [15], and the proposed model was proved to be complete and to account for the mean-reverting feature of the volatility process. This model is also non Markov as the past history $\left(V_{u}\right)_{u \in[t-\tau, t]}$ of the variance appears in its diffusion equation via the term $\ln \left(\frac{S_{t}}{S_{t-\tau}}\right)$, as it is shown in Swishchuk [23]. In the continuity of this approach, a series of papers were published by one of the authors focusing on the pricing of variance swaps in this delayed framework: one-factor 
stochastic volatility with delay has been presented in Swishchuk [23]; multifactor stochastic volatility with delay in Swishchuk [24]; one-factor stochastic volatility with delay and jumps in Swishchuk and $\mathrm{Li}$ [21]; and finally local Levy-based stochastic volatility with delay in Swishchuk and Malenfant [26].

Other papers related to the concept of delay are also of interest. For example, Kind et al. [17] obtained a diffusion approximation result for processes satisfying some equations with past dependent coefficients, with application to option pricing. Arriojas et al. [1] derived a Black\&Scholes formula for call options assuming the stock price follows a Stochastic Delay Differential Equation (SDDE). Mohammed and Bell have also published a series of papers in which they investigate various properties of SDDE (see e.g. [2], [3]).

Unfortunately, the model (3) doesn't lead to (semi-)closed formulas for the vanilla options, making it difficult to use for practitioners willing to calibrate on vanilla market prices. Nevertheless, one can notice that the Heston model and the delayed continuous-time GARCH model (3) are very similar in the sense that the expected values of the variances are the same - when we make the delay tend to 0 in (3). As mentioned before, the Heston framework is very convenient, and therefore it is naturally tempting to adjust the Heston dynamics in order to incorporate the delay introduced in (3). In this way, we considered in a first approach adjusting the Heston drift by a deterministic function of time so that the expected value of the variance under the delayed Heston model is equal to the one under the delayed GARCH model (3). In addition to making our delayed Heston framework coherent with (3), this construction makes the variance process diffusion dependent not on its past history $\left(V_{u}\right)_{u \in[t-\tau, t]}$, but on the past history of its risk-neutral expectation $\left(\mathbb{E}_{0}^{\mathbb{Q}}\left(V_{u}\right)\right)_{u \in[t-\tau, t]}$, preserving the Markov feature of the Heston model (where we denote $\mathbb{E}_{t}^{\mathbb{Q}}(\cdot):=\mathbb{E}^{\mathbb{Q}}\left(\cdot \mid \mathcal{F}_{t}\right)$ for some filtration $\left.\left(\mathcal{F}_{t}\right)_{t \geq 0}\right)$. The purpose of sections 2 and 3 is to present the Delayed Heston model as well as some calibration results on call option prices, with a comparison to the Heston model. In sections 4 and 5, we will consider the pricing and hedging of volatility and variance swaps in this model.

Volatility and variance swaps are contracts whose payoff depend (respectively convexly and linearly) on the realized variance of the underlying asset over some specified time interval. They provide pure exposure to volatility, and therefore make it a tradable market instrument. Variance Swaps are even considered by some practitioners to be vanilla derivatives. The most commonly traded variance swaps are discretely sampled and have a payoff $P_{n}^{V}(T)$ at maturity $T$ of the form:

$$
P_{n}^{V}(T)=N\left[\frac{252}{n} \sum_{i=0}^{n} \ln ^{2}\left(\frac{S_{i+1}}{S_{i}}\right)-K_{v a r}\right],
$$

where $S_{i}$ is the asset spot price on fixing time $t_{i} \in[0, T]$ (usually there is one fixing time each day, but there could be more, or less), $N$ the notional amount of the contract (in currency per unit of variance) and $K_{v a r}$ the strike specified 
in the contract. The corresponding volatility swap payoff $P_{n}^{v}(T)$ is given by:

$$
P_{n}^{v}(T)=N\left[\sqrt{\frac{252}{n} \sum_{i=0}^{n} \ln ^{2}\left(\frac{S_{i+1}}{S_{i}}\right)}-K_{v o l}\right] .
$$

One can also consider continuously sampled volatility and variance swaps (on which we will focus in this article), which payoffs are respectively defined as the limit when $n \rightarrow+\infty$ of their discretely sampled versions. Formally, if we denote $\left(V_{t}\right)_{t \geq 0}$ the stochastic volatility process of our asset, adapted to some brownian filtration $\left(\mathcal{F}_{t}\right)_{t>0}$, then the continuously-sampled realized variance $V_{R}$ from initiation date of the contract $t=0$ to maturity date $t=T$ is given by $V_{R}=\frac{1}{T} \int_{0}^{T} V_{s} d s$. The fair variance strike $K_{v a r}$ is calculated such that the initial value of the contract is 0 , and therefore is given by:

$$
\mathbb{E}_{0}^{\mathbb{Q}}\left[e^{-r T}\left(V_{R}-K_{\text {var }}\right)\right]=0 \Rightarrow K_{\text {var }}=\mathbb{E}_{0}^{\mathbb{Q}}\left(V_{R}\right) .
$$

In the same way, the fair volatility strike $K_{v o l}$ is given by:

$$
\mathbb{E}_{0}^{\mathbb{Q}}\left[e^{-r T}\left(\sqrt{V_{R}}-K_{v o l}\right)\right]=0 \Rightarrow K_{\text {vol }}=\mathbb{E}_{0}^{\mathbb{Q}}\left(\sqrt{V_{R}}\right) .
$$

The volatility swap fair strike might be difficult to compute explicitly as we have to compute the expectation of a square-root. In Brockhaus and Long [7], the following approximation - based on a Taylor expansion - was proposed to compute the expected value of the square-root of an almost surely non negative random variable $Z$ :

$$
\mathbb{E}(\sqrt{Z}) \approx \sqrt{\mathbb{E}(Z)}-\frac{\operatorname{Var}(Z)}{8 \mathbb{E}(Z)^{\frac{3}{2}}} .
$$

We will refer to this approximation in our paper as the Brockhaus\&Long approximation.

There exists a vast literature on volatility and variance swaps. We provide in the following lines a selection of papers covering important topics. Carr and Lee [8] provides an overview of the current market of volatility derivatives. They survey the early literature on the subject. They also provide relatively simple proofs of some fundamental results related to variance swaps and volatility swaps. Pricing of variance swaps for one-factor stochastic volatility is presented in Swishchuk [22]. Variance and volatility swaps in energy markets are considered in Swishchuk [25]. Broadie and Jain [6] covers pricing and dynamic hedging of volatility derivatives in the Heston model. Moreover, various papers deal with the VIX Index - the Chicago Board Options Exchange Market Volatility Index - which is a popular measure of the one month implied volatility on the S\&P 500 index (see e.g. Zhang and Zhu [27], Hao and Zhang [10] or Filipovic [9]).

The paper is organized as follows: in section 2, we present the Delayed Heston model; in section 3, we present calibration results (for underlying EURUSD on 12 dates ranging from Sep. $19^{\text {th }}$ to Oct. $17^{\text {th }} 20112011$ ) as well as a 
comparison with the Heston model. In section 4, we compute the price process $X_{t}(T):=\mathbb{E}_{t}^{\mathbb{Q}}\left(V_{R}\right)$ of the floating leg of the variance swap of maturity $T$, as well as the Brockhaus\&Long approximation of the price process $Y_{t}(T):=\mathbb{E}_{t}^{\mathbb{Q}}\left(\sqrt{V_{R}}\right)$ of the floating leg of the volatility swap of maturity $T$. This leads in particular to closed formulas for the fair volatility and variance strikes. In section 5 , we consider - in this model - dynamic hedging of volatility swaps using variance swaps.

\section{Presentation of the Delayed Heston model}

Throughout this paper, we will assume constant risk-free rate $r$, dividend yield $q$ and finite time-horizon $T$. We fix $(\Omega, \mathcal{F}, \mathbb{P})$ a probability space and we consider a stock whose price process is denoted by $\left(S_{t}\right)_{t \geq 0}$. We let $\mathbb{Q}$ be a riskneutral measure and we let $\left(Z_{t}^{\mathbb{Q}}\right)_{t \geq 0}$ and $\left(W_{t}^{\mathbb{Q}}\right)_{t \geq 0}$ be two correlated standard brownian motions on $(\Omega, \mathcal{F}, \mathbb{Q})$. We let the natural filtration associated to these brownian motions $\mathcal{F}_{t}:=\sigma\left(Z_{t}^{\mathbb{Q}}, W_{t}^{\mathbb{Q}}\right)$ and we denote $\mathbb{E}_{t}^{\mathbb{Q}}(\cdot):=\mathbb{E}^{\mathbb{Q}}\left(\cdot \mid \mathcal{F}_{t}\right)$ and $\operatorname{Var}_{t}^{\mathbb{Q}}(\cdot):=\operatorname{Var}^{\mathbb{Q}}\left(\cdot \mid \mathcal{F}_{t}\right)$.

We assume the following risk-neutral $\mathbb{Q}$ - stock price dynamics :

$$
d S_{t}=(r-q) S_{t} d t+S_{t} \sqrt{V_{t}} d Z_{t}^{\mathbb{Q}}
$$

The well-known Heston model has the following $\mathbb{Q}$-dynamics for the variance $V_{t}$ :

$$
d V_{t}=\gamma\left(\theta^{2}-V_{t}\right) d t+\delta \sqrt{V_{t}} d W_{t}^{\mathbb{Q}}
$$

where $\theta^{2}$ is the long-range variance, $\gamma$ the variance mean-reversion speed, $\delta$ the volatility of the variance and $\rho$ the brownian correlation coefficient $\left(\left\langle W^{\mathbb{Q}}, Z^{\mathbb{Q}}\right\rangle_{t}=\right.$ $\rho t)$. We also assume $S_{0}=s_{0}$ a.e. and $V_{0}=v_{0}$ a.e., for some positive constants $v_{0}, s_{0}$.

As explained in the introduction, the following delayed continuous-time GARCH dynamics have been introduced for the variance in Swishchuk [23]:

$$
\frac{d V_{t}}{d t}=\gamma \theta^{2}+\frac{\alpha}{\tau}\left[\int_{t-\tau}^{t} \sqrt{V_{s}} d Z_{s}^{\mathbb{Q}}-(\mu-r) \tau\right]^{2}-(\alpha+\gamma) V_{t},
$$

where $\mu$ stands for the real world $\mathbb{P}$-drift of the stock price $S_{t}$. We notice that $\theta^{2}$ (resp. $\gamma$ ) has been defined in introduction for the delayed continuous-time GARCH model as the value of the long-range variance (resp. variance meanreversion speed) when $\tau=0$, therefore it has the same meaning as the Heston long-range variance (resp. variance mean-reversion speed). That is why we use the same notations in both models.

We can see that the two models are very similar. Indeed, they both give the same expected value for $V_{t}$ as the delay goes to 0 in $(7)$, namely $\theta^{2}+\left(V_{0}-\right.$ 
$\left.\theta^{2}\right) e^{-\gamma t}$. The idea here is to adjust the Heston dynamics (6) in order to account for the delay introduced in (7). Our approach is to adjust the drift by a deterministic function of time so that the expected value of $V_{t}$ under the adjusted Heston model is the same as under (7). This approach can be seen as a correction by a pure delay term of amplitude $\alpha$ of the Heston drift by a deterministic function in order to account for the delay.

Namely, we assume the adjusted Heston dynamics:

$$
\begin{gathered}
d V_{t}=\left[\gamma\left(\theta^{2}-V_{t}\right)+\epsilon_{\tau}(t)\right] d t+\delta \sqrt{V_{t}} d W_{t}^{\mathbb{Q}}, \\
\epsilon_{\tau}(t):=\alpha \tau(\mu-r)^{2}+\frac{\alpha}{\tau} \int_{t-\tau}^{t} v_{s} d s-\alpha v_{t},
\end{gathered}
$$

with $v_{t}:=\mathbb{E}_{0}^{\mathbb{Q}}\left(V_{t}\right)$. It was shown in Swishchuk [23] that $v_{t}$ solves the following equation:

$$
\frac{d v_{t}}{d t}=\gamma \theta^{2}+\alpha \tau(\mu-r)^{2}+\frac{\alpha}{\tau} \int_{t-\tau}^{t} v_{s} d s-(\alpha+\gamma) v_{t},
$$

and that we have the following expression for $v_{t}$ :

$$
v_{t}=\theta_{\tau}^{2}+\left(V_{0}-\theta_{\tau}^{2}\right) e^{-\gamma_{\tau} t}
$$

with:

$$
\theta_{\tau}^{2}:=\theta^{2}+\frac{\alpha \tau(\mu-r)^{2}}{\gamma}
$$

By (11) and (15) (see below), we have $\lim _{t \rightarrow \infty} v_{t}=\theta_{\tau}^{2}$ and therefore the parameter $\theta_{\tau}^{2}$ can be interpreted as the adjusted value of the limit towards $v_{t}$ tends to as $t \rightarrow \infty$, that has been (positively) shifted from its original value $\theta^{2}$ because of the introduction of delay. We have $\theta_{\tau}^{2} \rightarrow \theta^{2}$ when $\tau \rightarrow 0$, which is coherent. We will see below that we can interpret the parameter $\gamma_{\tau}>0$ as the adjusted mean-reversion speed. This parameter is given in Swishchuk [23] by a (nonzero) solution to the following equation:

$$
\gamma_{\tau}=\alpha+\gamma+\frac{\alpha}{\gamma_{\tau} \tau}\left(1-e^{\gamma_{\tau} \tau}\right)
$$

By (9), (11) and (13) we get an explicit expression for the drift adjustment:

$$
\epsilon_{\tau}(t)=\alpha \tau(\mu-r)^{2}+\left(V_{0}-\theta_{\tau}^{2}\right)\left(\gamma-\gamma_{\tau}\right) e^{-\gamma_{\tau} t}
$$

The following simple property gives us some information about the correction term $\epsilon_{\tau}(t)$ and the parameter $\gamma_{\tau}$, that will be useful for interpretation purpose and in the derivation of the semi-closed formulas for call options in Appendix A. Indeed, given (15) and (11), the parameter $\gamma_{\tau}$ can be interpreted as the adjusted variance mean-reversion speed because it quantifies the speed at which $v_{t}$ tends to $\theta_{\tau}^{2}$ as $t \rightarrow \infty$, and we have by using a Taylor expansion in (13) that $\gamma_{\tau} \rightarrow \gamma$ when $\tau \rightarrow 0$, which is coherent. 
Property 1: $\gamma_{\tau}$ is the unique solution to (13) and:

$$
0<\gamma_{\tau}<\gamma, \quad \lim _{\tau \rightarrow 0} \sup _{t \in \mathbb{R}^{+}}\left|\epsilon_{\tau}(t)\right|=0 .
$$

Proof: Let's show $\gamma_{\tau} \geq 0$. If $\gamma_{\tau}<0$ then by (13) we have $\alpha+\gamma+\frac{\alpha}{\gamma_{\tau} \tau}\left(1-e^{\gamma_{\tau} \tau}\right)<0$, i.e. $1-e^{\gamma_{\tau} \tau}+\gamma_{\tau} \tau>-\frac{\gamma}{\alpha} \gamma_{\tau} \tau$. But $\tau>0$ so $\exists x_{0}>0$ s.t. $1-e^{-x_{0}}-x_{0}>\frac{\gamma}{\alpha} x_{0}$. A simple study shows that is impossible whenever $\frac{\gamma}{\alpha} \geq 0$, which is what we have by assumption. Therefore $\gamma_{\tau} \geq 0$, and in fact $\gamma_{\tau}>0$ since it is a nonzero solution of (13). If $\gamma \leq \gamma_{\tau}$ then by (13) $\gamma_{\tau} \tau+1-e^{\gamma_{\tau} \tau} \geq 0$. But $\gamma_{\tau} \tau>0$ therefore $\exists x_{0}>0$ s.t. $x_{0}+1-e^{x 0} \geq 0$. A simple study shows that is impossible. The uniqueness comes from a similar simple study. Now, because $\gamma_{\tau}>0$, we have $\sup _{t \in \mathbb{R}^{+}}\left|\epsilon_{\tau}(t)\right| \leq \alpha \tau(\mu-r)^{2}+\left|\left(V_{0}-\theta_{\tau}^{2}\right)\left(\gamma-\gamma_{\tau}\right)\right|$ and $\left(V_{0}-\theta_{\tau}^{2}\right)\left(\gamma-\gamma_{\tau}\right)=\circ(1)$ by (13). So $\lim _{\tau \rightarrow 0} \alpha \tau(\mu-r)^{2}+\left|\left(V_{0}-\theta_{\tau}^{2}\right)\left(\gamma-\gamma_{\tau}\right)\right|=0$.

Using (14) and (12), we can rewrite (8) as a time-dependent Heston model with time-dependent long-range variance $\tilde{\theta}_{t}^{2}$ :

$$
\begin{gathered}
d V_{t}=\gamma\left(\tilde{\theta}_{t}^{2}-V_{t}\right) d t+\delta \sqrt{V_{t}} d W_{t}^{\mathbb{Q}}, \\
\tilde{\theta}_{t}^{2}:=\theta_{\tau}^{2}+\left(V_{0}-\theta_{\tau}^{2}\right) \frac{\left(\gamma-\gamma_{\tau}\right)}{\gamma} e^{-\gamma_{\tau} t} .
\end{gathered}
$$

The parameter $\theta_{\tau}^{2}$ is - as we mentioned above - the adjusted value of the limit towards which $v_{t}$ tends to as $t \rightarrow \infty$. For this reason, it is coherent that it is also the limiting value of the time-dependent long-range variance $\tilde{\theta}_{t}^{2}$ as $t \rightarrow \infty$ (by (17) and (15)).

\section{Calibration on call option prices and comparison to the Heston model}

Following Kahl and Jäckel [13] and Mikhailov and Noegel [20], it is possible to get semi-closed formulas for call options in our delayed Heston model. Indeed, our model is a time-dependent Heston model with time-dependent long-range variance $\tilde{\theta}_{t}^{2}$. We refer to Appendix A for the procedure to derive such semiclosed formulas.

We perform our calibration on September $30^{\text {th }} 2011$ for underlying EURUSD on the whole volatility surface (maturities from 1M to 10Y, strikes ATM, 25D Call/Put, 10D Call/Put). The implied volatility surface, the Zero Coupon curves EUR Vs. Euribor 6M and USD Vs. Libor 3M and the spot price are taken from Bloomberg (mid prices). The drift $\mu=0.0188$ is estimated from $7.5 \mathrm{Y}$ of daily close prices (source: www.forexrate.co.uk).

The calibration procedure is a least-squares minimization procedure that we perform via MATLAB (function lsqnonlin that uses a trust-region-reflective 
algorithm). The Heston integral (83) is computed via the MATLAB function quadl that uses a recursive adaptive Lobatto quadrature. The integral $\int_{0}^{t} e^{-\gamma_{\tau} s} D(s, u) d s$ in $(81)$ is computed via a composite Simpson's rule with 100 points.

The calibrated parameters for delayed Heston are:

$$
\left(V_{0}, \gamma, \theta^{2}, \delta, \rho, \alpha, \tau\right)=\left(0.0343,3.9037,10^{-8}, 0.808,-0.5057,71.35,0.7821\right),
$$

and for Heston, they are:

$$
\left(V_{0}, \gamma, \theta^{2}, \delta, \rho\right)=(0.0328,0.5829,0.0256,0.3672,-0.4824) .
$$

We notice that we cannot compare straightforwardly the parameters $\theta^{2}$ of both models. Indeed, as mentioned above, the Delayed Heston model has a timedependent long range variance $\tilde{\theta}_{t}^{2}$ which has been shifted away from its original value $\theta^{2}$ because of the introduction of the delay $\tau$. When $\tau=0, \tilde{\theta}_{t}^{2}=\theta^{2}$ but when $\tau>0, \tilde{\theta}_{t}^{2}$ and $\theta^{2}$ differ. Therefore, to be coherent, one should compare the Heston long range-variance $\theta^{2}=0.0256$ with the Delayed Heston timedependent long-range variance $\tilde{\theta}_{t}^{2}$. Below we give the value of $\tilde{\theta}_{t}^{2}$ for different maturities $t$ :

\begin{tabular}{|c|c|}
\hline Maturity & $\tilde{\theta}_{t}^{2}$ \\
\hline $1 \mathrm{M}$ & 0.0325 \\
\hline $2 \mathrm{M}$ & 0.0322 \\
\hline $3 \mathrm{M}$ & 0.0319 \\
\hline $6 \mathrm{M}$ & 0.031 \\
\hline $1 \mathrm{Y}$ & 0.0294 \\
\hline $2 \mathrm{Y}$ & 0.0364 \\
\hline $5 \mathrm{Y}$ & 0.0184 \\
\hline $10 \mathrm{Y}$ & 0.0102 \\
\hline
\end{tabular}

Table 1: Parameter $\tilde{\theta}_{t}^{2}$ for different maturities $t$.

We remark that the short and medium term values (less than $2 \mathrm{Y}$ ) of $\tilde{\theta}_{t}^{2}$ are similar to the value of $\theta^{2}$ in the Heston model, but that for long maturities, the value of $\tilde{\theta}_{t}^{2}$ decreases significantly. Allowing this time-dependence of the the long-range variance could be an explanation why the Delayed Heston model outperforms the Heston model especially for long maturities (see the discussion below). Similarly, the Heston mean-reversion speed $\gamma=0.58$ has to be compared with the Delayed Heston adjusted mean-reversion speed $\gamma_{\tau}$, which is given by (13) and is approximately equal to 0.12 on our calibration date. Focusing on the delay parameters $\alpha$ and $\tau$, they were expected to be significantly non zero because as we will see below, the Delayed Heston model significantly outperforms the Heston model in terms of calibration error (and standard deviation of the calibration errors): if $\alpha$ and $\tau$ were close to 0 , the calibration errors would have been approximately the same for both models, because again, the Delayed Heston model reduces to the Heston model when the delay term vanishes, i.e. when $\tau=0$ or $\alpha=0$.

The calibration errors for all call options (expressed as the absolute value of the difference between market and model implied Black \& Scholes volatilities, in 
bp) for the Heston model and our Delayed Heston model are given below. The results show a $44 \%$ reduction of the average absolute calibration error (46bp for delayed Heston, 81bp for Heston). It is to be noted that we didn't use any weight matrix in our calibration procedure, i.e. the calibration aims at minimizing the sum of the (squares of the) errors of each call option, equally weighted. In practice, one might be willing to give more importance to ATM options for instance, or options of a certain range of maturities. The optimization algorithm aims at minimizing the sum of the squares of the errors: in other words, it aims at minimizing the average absolute calibration error. For this reason, it might be the case that for some specific option (e.g. ATM 6M, see table below), the Heston model has a lower model error than the Delayed Heston model. But the total calibration error for the Delayed Heston model is always expected to be lower than for the Heston model.

On our calibration date, the Delayed Heston model seems to outperform the Heston model specifically for long maturities $(\geq 3 \mathrm{Y})$ : if we consider only these options, the average absolute error is of $79 \mathrm{bp}$ for the Heston model and 33bp for the Delayed Heston model, which represents a $58 \%$ reduction of the calibration error. We can also note that for ATM options only, the improvement is significant too (43bp Vs. 92bp, i.e. an error reduction of 54\%). For medium maturity options (6M to $2 \mathrm{Y}$ ), the Delayed Heston model still outperforms the Heston model but less significantly (53bp Vs. $75 \mathrm{bp}$, i.e. an error reduction of $30 \%$ ), and we have the same observation for very out of the money options (10 Delta Call and Put, 51bp Vs. 79bp, i.e. an error reduction of $35 \%$ ).

Another very interesting observation we can make is that the standard deviation of the calibration errors is much lower for the Delayed Heston model compared to the Heston model (34bp Vs. 52bp, which represents a $35 \%$ reduction of the standard deviation): in addition to improving the average absolute calibration error, it also improves the distance of the individual errors to the average error, which is highly appreciable in practice because it means that you won't face the case where some options are priced really poorly by the model whereas some others are priced almost perfectly.

\begin{tabular}{|c|c|c|c|c|c|}
\hline & ATM & $25 \mathrm{D}$ & Call 25D Put & $10 \mathrm{D}$ & Call 10D Put \\
\hline $1 \mathrm{M}$ & 152 & 192 & 41 & 193 & 67 \\
\hline $2 \mathrm{M}$ & 114 & 139 & 15 & 136 & 81 \\
\hline $3 \mathrm{M}$ & 89 & 109 & 3 & 110 & 92 \\
\hline $4 \mathrm{M}$ & 48 & 61 & 17 & 67 & 101 \\
\hline $6 \mathrm{M}$ & 5 & 15 & 34 & 29 & 85 \\
\hline $9 \mathrm{M}$ & 59 & 42 & 63 & 2 & 85 \\
\hline $1 \mathrm{Y}$ & 107 & 83 & 102 & 31 & 96 \\
\hline $1.5 \mathrm{Y}$ & 141 & 116 & 111 & 42 & 73 \\
\hline $2 \mathrm{Y}$ & 166 & 137 & 127 & 54 & 68 \\
\hline $3 Y$ & 145 & 124 & 77 & 52 & 0 \\
\hline $4 Y$ & 96 & 95 & 18 & 37 & 66 \\
\hline $5 \mathrm{Y}$ & 29 & 47 & 52 & 7 & 138 \\
\hline $7 \mathrm{Y}$ & 39 & 10 & 112 & 28 & 186 \\
\hline $10 \mathrm{Y}$ & 100 & 67 & 168 & 58 & 225 \\
\hline
\end{tabular}

Table 2: Heston Absolute Calibration Error (in bp of the Black 8 Scholes volatility). 


\begin{tabular}{|c|c|c|c|c|c|}
\hline & ATM & $25 \mathrm{D}$ & Call 25D Put & $10 \mathrm{D}$ & Call 10D Put \\
\hline $1 \mathrm{M}$ & 116 & 91 & 109 & 128 & 115 \\
\hline $2 \mathrm{M}$ & 44 & 24 & 59 & 54 & 88 \\
\hline $3 \mathrm{M}$ & 14 & 3 & 32 & 36 & 60 \\
\hline $4 \mathrm{M}$ & 18 & 28 & 1 & 5 & 29 \\
\hline $6 \mathrm{M}$ & 31 & 37 & 23 & 19 & 3 \\
\hline $9 \mathrm{M}$ & 45 & 45 & 56 & 37 & 57 \\
\hline $1 \mathrm{Y}$ & 51 & 47 & 82 & 50 & 104 \\
\hline $1.5 \mathrm{Y}$ & 29 & 30 & 79 & 49 & 129 \\
\hline $2 \mathrm{Y}$ & 24 & 23 & 83 & 47 & 139 \\
\hline $3 Y$ & 11 & 9 & 29 & 30 & 90 \\
\hline $4 Y$ & 41 & 28 & 14 & 17 & 38 \\
\hline $5 \mathrm{Y}$ & 76 & 55 & 59 & 5 & 16 \\
\hline $7 \mathrm{Y}$ & 71 & 49 & 58 & 1 & 14 \\
\hline $10 \mathrm{Y}$ & 26 & 8 & 18 & 47 & 24 \\
\hline
\end{tabular}

Table 3: Delayed Heston Absolute Calibration Error (in bp of the Black 8 Scholes volatility).

\begin{tabular}{|l|c|c|}
\hline & Delayed Heston & Heston \\
\hline $\begin{array}{l}\text { Standard Deviation of } \\
\text { the calibration errors } \\
\text { (bp) }\end{array}$ & $33.67(35 \%)$ & 52.12 \\
\hline
\end{tabular}

Table 4: Standard Deviation of the calibration errors in $\mathrm{bp}$. The reduction of this error is indicated in brackets.

In order to i) check that our calibration on September $30^{\text {th }} 2011$ was not an exception and ii) investigate the stability of the calibrated parameters, we performed calibrations on 11 additional dates evenly spaced around September $30^{\text {th }}$ 2011, ranging from September 19th 2011 to October 17th 2011. We chose a one month window because from the past experience of the authors in the financial industry, it can happen that the parameters are recalibrated by financial institutions every month only, and not every day (because it would be too time-consuming) and therefore the choice of a one month window seems reasonable to investigate the stability of the parameters. We summarize the findings in the tables below. We find that the Delayed Heston model always outperforms significantly the Heston model (average calibration error reduction varying from $29 \%$ to $56 \%$ ), and that the Delayed Heston model is performant especially for long maturities $(\geq 3 \mathrm{Y}$, calibration error reduction varying from $40 \%$ to $66 \%$ ) and ATM options (calibration error reduction varying from $42 \%$ to $67 \%$ ). Finally, the standard deviation of the calibrations errors is always reduced significantly by the Delayed Heston model (reduction varying from $23 \%$ to $49 \%)$.

\begin{tabular}{|l|c|c|c|c|c|c|c|c|c|c|c|c|}
\hline Date (2011) & Sep. 19 & ep. 21 & Sep. 23 & Sep. 27 & Sep. 29 & Oct. 3 & Oct. 5 & Oct. 7 & Oct. 11 & Oct. 13 & Oct. 17 \\
\hline $\begin{array}{l}\text { Total Error Re- } \\
\text { duction (\%) }\end{array}$ & 44 & 45 & 56 & 47 & 38 & 51 & 42 & 37 & 38 & 29 & 39 \\
\hline $\begin{array}{l}\text { Long Maturity } \\
\begin{array}{l}\text { Error Reduction } \\
(\%)\end{array}\end{array}$ & 58 & 63 & 65 & 55 & 53 & 66 & 55 & 51 & 48 & 40 & 59 \\
\hline $\begin{array}{l}\text { ATM Error Re- } \\
\text { duction (\%) }\end{array}$ & 57 & 55 & 67 & 62 & 55 & 65 & 56 & 51 & 50 & 42 & 53 \\
\hline
\end{tabular}

Table 5: Summary of the calibration error reductions. 


\begin{tabular}{|l|c|c|c|c|c|c|c|c|c|c|c|c|}
\hline Date (2011) & Sep. 19 & Sep. 21 & Sep. 23 & Sep. 27 & Sep. 29 & Oct. 3 & Oct. 5 & Oct. 7 & Oct. 11 & Oct. 13 & Oct. 17 \\
\hline $\begin{array}{l}\text { Calibration er- } \\
\text { rors St. Dev. } \\
\text { Reduction (\%) }\end{array}$ & 43 & 45 & 49 & 46 & 31 & 49 & 40 & 29 & 29 & 23 & 29 \\
\hline
\end{tabular}

Table 6: Summary of the calibration errors St. Dev reductions.

In order to investigate the stability of the model parameters, we present below the calibrated parameters for the Heston model and the Delayed Heston model from September $19^{\text {th }} 2011$ to October $17^{\text {th }} 2011$.

\begin{tabular}{|l|c|c|c|c|c|c|c|c|c|c|c|c|}
\hline Date (2011) & Sep. 19 & eep. 21 & Sep. 23 & Sep. 27 & Sep. 29 & Oct. 3 & Oct. 5 & Oct. 7 & Oct. 11 & Oct. 13 & Oct. 17 \\
\hline$V_{0}$ & 0.0313 & 0.0337 & 0.0384 & 0.0354 & 0.0335 & 0.0368 & 0.0344 & 0.0295 & 0.0279 & 0.0271 & 0.0283 \\
\hline$\gamma$ & 3.99 & 3.72 & 3.82 & 3.72 & 4.52 & 3.47 & 3.86 & 3.71 & 3.13 & 3.08 & 3.39 \\
\hline$\theta^{2}$ & $5 \mathrm{e}-4$ & $7 \mathrm{e}-6$ & $2 \mathrm{e}-4$ & $1 \mathrm{e}-8$ & $1 \mathrm{e}-8$ & $1 \mathrm{e}-5$ & $3 \mathrm{e}-4$ & $2 \mathrm{e}-3$ & $1 \mathrm{e}-3$ & $5 \mathrm{e}-3$ & $4 \mathrm{e}-3$ \\
\hline$\delta$ & 0.79 & 0.75 & 0.82 & 0.81 & 0.89 & 0.78 & 0.81 & 0.76 & 0.68 & 0.67 & 0.80 \\
\hline$\rho$ & -0.51 & -0.51 & -0.52 & -0.50 & -0.49 & -0.51 & -0.51 & -0.51 & -0.51 & -0.51 & -0.49 \\
\hline$\alpha$ & 82.2 & 77.5 & 64.5 & 166.7 & 124 & 66.6 & 76.5 & 90.2 & 125.1 & 83.7 & 77.3 \\
\hline$\tau$ & 0.86 & 0.77 & 0.71 & 0.32 & 0.59 & 0.72 & 0.78 & 0.90 & 0.67 & 1.00 & 0.81 \\
\hline
\end{tabular}

Table 7: Calibrated Parameters for the Delayed Heston model.

\begin{tabular}{|l|c|c|c|c|c|c|c|c|c|c|c|c|}
\hline Date (2011) & Sep. 19 & Sep. 21 & Sep. 23 & Sep. 27 & Sep. 29 & Oct. 3 & Oct. 5 & Oct. 7 & Oct. 11 & Oct. 13 & Oct. 17 \\
\hline$V_{0}$ & 0.0298 & 0.0322 & 0.0369 & 0.0338 & 0.0311 & 0.0351 & 0.0326 & 0.0283 & 0.0271 & 0.0262 & 0.0269 \\
\hline$\gamma$ & 0.54 & 0.46 & 0.45 & 0.43 & 0.35 & 0.44 & 0.43 & 0.89 & 1.22 & 1.13 & 0.92 \\
\hline$\theta^{2}$ & 0.0258 & 0.026 & 0.0258 & 0.0264 & 0.0276 & 0.0265 & 0.0275 & 0.0265 & 0.0249 & 0.0254 & 0.024 \\
\hline$\delta$ & 0.34 & 0.33 & 0.35 & 0.35 & 0.32 & 0.34 & 0.34 & 0.41 & 0.45 & 0.43 & 0.39 \\
\hline$\rho$ & -0.49 & -0.49 & -0.49 & -0.48 & -0.48 & -0.50 & -0.49 & -0.50 & -0.50 & -0.49 & -0.51 \\
\hline
\end{tabular}

Table 8: Calibrated Parameters for the Heston model.

We can see that in average, the parameters stay relatively stable throughout this 1 month time window. In this case, it would be reasonable to use the same parameters throughout the 1 month time window as some financial institutions do (from the past experience of the authors in the financial industry). Of course, there are some periods of high volatility in which not recalibrating the model parameters often enough might lead to a significant mispricing of the call options by the model.

\section{Pricing Variance and Volatility Swaps}

In this section, we derive a closed formula for the Brockhaus\&Long approximation of the volatility swap price using the change of time method introduced in Swishchuk [22], as well as the price of the variance swap. Precisely, in Brockhaus and Long [7], the following approximation was presented to compute the expected value of the square-root of an almost surely non negative random variable $Z: \mathbb{E}(\sqrt{Z}) \approx \sqrt{\mathbb{E}(Z)}-\frac{\operatorname{Var}(Z)}{8 \mathbb{E}(Z)^{\frac{3}{2}}}$. We denote $V_{R}:=\frac{1}{T} \int_{0}^{T} V_{s} d s$ the realized variance on $[0, T]$. 
We let $X_{t}(T):=\mathbb{E}_{t}^{\mathbb{Q}}\left(V_{R}\right)$ (resp. $Y_{t}(T):=\mathbb{E}_{t}^{\mathbb{Q}}\left(\sqrt{V_{R}}\right)$ ) the price process of the floating leg of the variance swap (resp. volatility swap) of maturity $T$.

Theorem 1: The price process $X_{t}(T)$ of the floating leg of the variance swap of maturity $T$ in the delayed Heston model (5)-(8) is given by:

$$
\begin{aligned}
X_{t}(T)= & \frac{1}{T} \int_{0}^{t} V_{s} d s+\frac{T-t}{T} \theta_{\tau}^{2}+\left(V_{t}-\theta_{\tau}^{2}\right)\left(\frac{1-e^{-\gamma(T-t)}}{\gamma T}\right) \\
& +\left(V_{0}-\theta_{\tau}^{2}\right) e^{-\gamma_{\tau} t}\left(\frac{1-e^{-\gamma_{\tau}(T-t)}}{\gamma_{\tau} T}-\frac{1-e^{-\gamma(T-t)}}{\gamma T}\right) .
\end{aligned}
$$

Proof: By definition, $X_{t}(T)=\mathbb{E}_{t}^{\mathbb{Q}}\left(\frac{1}{T} \int_{0}^{T} V_{s} d s\right)=\frac{1}{T} \int_{0}^{t} V_{s} d s+\frac{1}{T} \int_{t}^{T} \mathbb{E}_{t}^{\mathbb{Q}}\left(V_{s}\right) d s$. In the previous integral, the interchange between the expectation and the integral is justified by the use of Tonelli's theorem, as the variance process $(t, \omega) \rightarrow V_{t}(\omega)$ is a.e. nonnegative and measurable. Let $s \geq t$. Then we have by (8) that $\mathbb{E}_{t}^{\mathbb{Q}}\left(V_{s}-V_{t}\right)=\mathbb{E}_{t}^{\mathbb{Q}}\left(V_{s}\right)-$ $V_{t}=\int_{t}^{s} \gamma\left(\theta^{2}-\mathbb{E}_{t}^{\mathbb{Q}}\left(V_{u}\right)\right)+\epsilon_{\tau}(u) d u+\mathbb{E}_{t}^{\mathbb{Q}}\left(\int_{t}^{s} \sqrt{V_{u}} d W_{u}^{\mathbb{Q}}\right)$. Again, the interchange of the expectation and the integral $\mathbb{E}_{t}^{\mathbb{Q}}\left(\int_{t}^{s} \gamma\left(\theta^{2}-V_{u}\right)+\epsilon_{\tau}(u) d u\right)=\int_{t}^{s} \gamma\left(\theta^{2}-\mathbb{E}_{t}^{\mathbb{Q}}\left(V_{u}\right)\right)+\epsilon_{\tau}(u) d u$ is obtained the following way:

$$
\mathbb{E}_{t}^{\mathbb{Q}}\left(\int_{t}^{s} \gamma\left(\theta^{2}-V_{u}\right)+\epsilon_{\tau}(u) d u\right)=\int_{t}^{s} \gamma \theta^{2}+\epsilon_{\tau}(u) d u-\gamma \mathbb{E}_{t}^{\mathbb{Q}}\left(\int_{t}^{s} V_{u} d u\right) .
$$

Then again, by Tonelli's theorem we get $\mathbb{E}_{t}^{\mathbb{Q}}\left(\int_{t}^{s} V_{u} d u\right)=\int_{t}^{s} \mathbb{E}_{t}^{\mathbb{Q}}\left(V_{u}\right) d u$, which justifies the interchange.

Now, $\left(\sqrt{V_{t}}\right)_{t \geq 0}$ is an adapted process (to our filtration $\left.\left(\mathcal{F}_{t}\right)_{t \geq 0}\right)$ s.t. $\mathbb{E}^{\mathbb{Q}}\left(\int_{0}^{T} V_{u} d u\right)=$ $\int_{0}^{T} \mathbb{E}^{\mathbb{Q}}\left(V_{u}\right) d u<+\infty$ (by Tonelli's theorem), therefore $\int_{0}^{t} \sqrt{V_{u}} d W_{u}^{\mathbb{Q}}$ is a martingale and we have $\mathbb{E}_{t}^{\mathbb{Q}}\left(\int_{t}^{s} \sqrt{V_{u}} d W_{u}^{\mathbb{Q}}\right)=0$. Therefore $\forall s \geq t \geq 0$, the function $s \rightarrow \mathbb{E}_{t}^{\mathbb{Q}}\left(V_{s}\right)$ is a solution of $y_{s}^{\prime}=\gamma\left(\theta^{2}-y_{s}\right)+\epsilon_{\tau}(s)$ with initial condition $y_{t}=V_{t}$. This gives us $\mathbb{E}_{t}^{\mathbb{Q}}\left(V_{s}\right)=\theta_{\tau}^{2}+\left(V_{t}-\theta_{\tau}^{2}\right) e^{-\gamma(s-t)}+\left(V_{0}-\theta_{\tau}^{2}\right) e^{-\gamma_{\tau} t}\left(e^{-\gamma_{\tau}(s-t)}-e^{-\gamma(s-t)}\right)$. Integrating the latter in the variable $s$ via $\int_{t}^{T} \mathbb{E}_{t}^{\mathbb{Q}}\left(V_{s}\right) d s$ completes the proof.

Corollary 1: The price $K_{v a r}$ of the variance swap of maturity $T$ at initiation of the contract $t=0$ in the delayed Heston model (5)-(8) is given by:

$$
K_{v a r}=\theta_{\tau}^{2}+\left(V_{0}-\theta_{\tau}^{2}\right) \frac{1-e^{-\gamma_{\tau} T}}{\gamma_{\tau} T} .
$$

Proof: By definition, $K_{v a r}=X_{0}(T)$.

Now, let:

$$
x_{t}:=-\left(V_{0}-\theta_{\tau}^{2}\right) e^{\left(\gamma-\gamma_{\tau}\right) t}+e^{\gamma t}\left(V_{t}-\theta_{\tau}^{2}\right) .
$$

Then by Ito's Lemma we get:

$$
d x_{t}=\delta e^{\gamma t} \sqrt{\left(x_{t}+\left(V_{0}-\theta_{\tau}^{2}\right) e^{\left(\gamma-\gamma_{\tau}\right) t}\right) e^{-\gamma t}+\theta_{\tau}^{2}} d W_{t}^{\mathbb{Q}} .
$$


Which is of the form $d x_{t}=f\left(t, x_{t}\right) d W_{t}^{\mathbb{Q}}$ with:

$$
f(t, x):=\delta e^{\gamma t} \sqrt{\left(x+\left(V_{0}-\theta_{\tau}^{2}\right) e^{\left(\gamma-\gamma_{\tau}\right) t}\right) e^{-\gamma t}+\theta_{\tau}^{2}} .
$$

Indeed, since $x_{t}=g\left(t, V_{t}\right)$ with $g(t, x):=-\left(V_{0}-\theta_{\tau}^{2}\right) e^{\left(\gamma-\gamma_{\tau}\right) t}+e^{\gamma t}\left(x-\theta_{\tau}^{2}\right)$, the multidimensional version of Ito's lemma reads:

$$
d x_{t}=d g\left(t, V_{t}\right)=g_{t}\left(t, V_{t}\right) d t+g_{x}\left(t, V_{t}\right) d V_{t}+\frac{1}{2} g_{x x}\left(t, V_{t}\right) d\langle V, V\rangle_{t},
$$

where $\langle V, V\rangle_{t}$ is the quadratic variation of the process $\left(V_{t}\right)_{t \geq 0}$ (see e.g. [14], theorem 3.6. of section 3.3). Since $g_{x x}(t, x)=0, g_{t}\left(t, V_{t}\right)=-\left(\gamma-\gamma_{\tau}\right)\left(V_{0}-\right.$ $\left.\theta_{\tau}^{2}\right) e^{\left(\gamma-\gamma_{\tau}\right) t}+\gamma e^{\gamma t}\left(V_{t}-\theta_{\tau}^{2}\right)$ and $g_{x}\left(t, V_{t}\right)=e^{\gamma t}$, we get, using (8), (12) and (14):

$$
\begin{aligned}
d x_{t}= & g_{t}\left(t, V_{t}\right) d t+g_{x}\left(t, V_{t}\right) d V_{t} \\
= & -\left(\gamma-\gamma_{\tau}\right)\left(V_{0}-\theta_{\tau}^{2}\right) e^{\left(\gamma-\gamma_{\tau}\right) t} d t+\gamma e^{\gamma t}\left(V_{t}-\theta_{\tau}^{2}\right) d t+e^{\gamma t} d V_{t} \\
= & -e^{\gamma t}\left(\epsilon_{\tau}(t)-\gamma\left(\theta_{\tau}^{2}-\theta^{2}\right)\right) d t+\gamma e^{\gamma t}\left(V_{t}-\theta_{\tau}^{2}\right) d t \\
& +e^{\gamma t}\left[\gamma\left(\theta^{2}-V_{t}\right)+\epsilon_{\tau}(t)\right] d t+e^{\gamma t} \delta \sqrt{V_{t}} d W_{t}^{\mathbb{Q}} \\
= & e^{\gamma t} \delta \sqrt{V_{t}} d W_{t}^{\mathbb{Q}} .
\end{aligned}
$$

The fact that $V_{t}=\left(x_{t}+\left(V_{0}-\theta_{\tau}^{2}\right) e^{\left(\gamma-\gamma_{\tau}\right) t}\right) e^{-\gamma t}+\theta_{\tau}^{2}$ by definition of $x_{t}(21)$ completes the proof.

Because $d x_{t}=f\left(t, x_{t}\right) d W_{t}^{\mathbb{Q}}$, the process $\left(x_{t}\right)_{t \geq 0}$ is a continuous local martingale, and even a true martingale since $\mathbb{E}^{\mathbb{Q}}\left(\int_{0}^{T} f^{2}\left(s, x_{s}\right) d s\right)=\int_{0}^{T} \mathbb{E}^{\mathbb{Q}}\left(f^{2}\left(s, x_{s}\right)\right) d s<$ $\infty$ (again, the interchange between expectation and integral follows from Tonelli's theorem). We can use the change of time method introduced in Swishchuk [22] and we get $x_{t}=\tilde{W}_{\phi_{t}}$, where $\tilde{W}_{t}$ is a $\mathcal{F}_{\phi_{t}^{-1}}-$ adapted $\mathbb{Q}$-Brownian motion, which is based on the fact that every continuous local martingale can be represented as a time-changed brownian motion. The process $\left(\phi_{t}\right)_{t>0}$ is a.e. increasing, non negative, $\mathcal{F}_{t}$ - adapted and is called the change of time process. This process is also equal to the quadratic variation $\langle x\rangle_{t}$ of the (square-integrable) continuous martingale $x_{t}$ (see [14], section 3.2, Proposition 2.10.).

Expressions of $\phi_{t}, \phi_{t}^{-1}$ and $\tilde{W}_{t}$ are given by:

$$
\begin{gathered}
\phi_{t}=\langle x\rangle_{t}=\int_{0}^{t} f^{2}\left(s, x_{s}\right) d s, \\
\tilde{W}_{t}=\int_{0}^{\phi_{t}^{-1}} f\left(s, x_{s}\right) d W_{s}^{\mathbb{Q}}, \\
\phi_{t}^{-1}=\int_{0}^{t} \frac{1}{f^{2}\left(\phi_{s}^{-1}, x_{\phi_{s}^{-1}}\right)} d s .
\end{gathered}
$$


To see that $\phi_{t}^{-1}$ has the following form, observe that:

$$
\phi_{\phi_{t}}^{-1}=\int_{0}^{\phi_{t}} \frac{1}{f^{2}\left(\phi_{s}^{-1}, x_{\phi_{s}^{-1}}\right)} d s .
$$

Now make the change of variable $s=\phi_{u}$, so that $d s=d \phi_{u}=f^{2}\left(u, x_{u}\right) d u$. We get:

$$
\phi_{\phi_{t}}^{-1}=\int_{0}^{t} \frac{f^{2}\left(u, x_{u}\right)}{f^{2}\left(\phi_{\phi_{u}}^{-1}, x_{\phi_{\phi_{u}}^{-1}}\right)} d u=\int_{0}^{t} \frac{f^{2}\left(u, x_{u}\right)}{f^{2}\left(u, x_{u}\right)} d u=t .
$$

This immediately yields:

$$
V_{t}=\theta_{\tau}^{2}+\left(V_{0}-\theta_{\tau}^{2}\right) e^{-\gamma_{\tau} t}+e^{-\gamma t} \tilde{W}_{\phi_{t}} .
$$

Lemma 1: For $s, t \geq 0$ we have:

$$
\mathbb{E}_{t}^{\mathbb{Q}}\left(\tilde{W}_{\phi_{s}}\right)=\tilde{W}_{\phi_{t \wedge s}}
$$

and for $s, u \geq t$ :

$$
\begin{aligned}
\mathbb{E}_{t}^{\mathbb{Q}}\left(\tilde{W}_{\phi_{s}} \tilde{W}_{\phi_{u}}\right) & =x_{t}^{2}+\delta^{2}\left[\theta_{\tau}^{2}\left(\frac{e^{2 \gamma(s \wedge u)}-e^{2 \gamma t}}{2 \gamma}\right)\right. \\
& \left.+\left(V_{0}-\theta_{\tau}^{2}\right)\left(\frac{e^{\left(2 \gamma-\gamma_{\tau}\right)(s \wedge u)}-e^{\left(2 \gamma-\gamma_{\tau}\right) t}}{2 \gamma-\gamma_{\tau}}\right)+x_{t}\left(\frac{e^{\gamma(s \wedge u)}-e^{\gamma t}}{\gamma}\right)\right] .
\end{aligned}
$$

Proof: (36) comes from the fact that $x_{t}=\tilde{W}_{\phi_{t}}$ is a martingale. Let $s \geq u \geq t$. Then by iterated conditioning: $\underset{\tilde{E}}{\mathbb{Q}}\left(\tilde{W}_{\phi_{s}} \tilde{W}_{\phi_{u}}\right)=\mathbb{E}_{t}^{\mathbb{Q}}\left(\mathbb{E}_{u}^{\mathbb{Q}}\left(\tilde{W}_{\phi_{s}} \tilde{W}_{\phi_{u}}\right)\right)=\mathbb{E}_{t}^{\mathbb{Q}}\left(\tilde{W}_{\phi_{u}} \mathbb{E}_{u}^{\mathbb{Q}}\left(\tilde{W}_{\phi_{s}}\right)\right)=$ $\mathbb{E}_{t}^{\mathbb{Q}}\left(\tilde{W}_{\phi_{u}}^{2}\right)$, because $x_{t}=\tilde{W}_{\phi_{t}}$ is a martingale. Now, by definition of the quadratic variation, $x_{u}^{2}-\langle x\rangle_{u}$ is a martingale and therefore $\mathbb{E}_{t}^{\mathbb{Q}}\left(\tilde{W}_{\phi_{u}}^{2}\right)=x_{t}^{2}-\langle x\rangle_{t}+\mathbb{E}_{t}^{\mathbb{Q}}\left(\langle x\rangle_{u}\right)=$ $x_{t}^{2}-\phi_{t}+\mathbb{E}_{t}^{\mathbb{Q}}\left(\phi_{u}\right)=x_{t}^{2}-\phi_{t}+\phi_{t}+\mathbb{E}_{t}^{\mathbb{Q}}\left(\int_{t}^{u} f^{2}\left(s, x_{s}\right) d s\right)$. We can again interchange expectation and integral by Tonelli's theorem. By definition of $f^{2}\left(s, x_{s}\right)$ (the latter is a linear function of $x_{s}$ ) and since $x_{t}$ martingale, then we have (for $s \geq t$ ) $\mathbb{E}_{t}^{\mathbb{Q}}\left(f^{2}\left(s, x_{s}\right)\right)=f^{2}\left(s, x_{t}\right)$, and therefore $\mathbb{E}_{t}^{\mathbb{Q}}\left(\tilde{W}_{\phi_{s}} \tilde{W}_{\phi_{u}}\right)=x_{t}^{2}+\int_{t}^{u} f^{2}\left(s, x_{t}\right) d s$. We use the fact that, by definition of $f$ in (23):

$$
f^{2}\left(s, x_{t}\right)=\delta^{2} e^{2 \gamma s}\left[\left(x_{t}+\left(V_{0}-\theta_{\tau}^{2}\right) e^{\left(\gamma-\gamma_{\tau}\right) s}\right) e^{-\gamma s}+\theta_{\tau}^{2}\right],
$$

to integrate the latter expression with respect to $s$ to complete the proof.

The following theorem gives the expression of the Brockhaus\&Long approximation of the volatility swap floating leg price process $Y_{t}(T)$. 
Theorem 2: The Brockhaus\&Long approximation of the price process $Y_{t}(T)$ of the floating leg of the volatility swap of maturity $T$ in the delayed Heston model (5)-(8) is given by:

$$
Y_{t}(T) \approx \sqrt{X_{t}(T)}-\frac{\operatorname{Var}_{t}^{\mathbb{Q}}\left(V_{R}\right)}{8 X_{t}(T)^{\frac{3}{2}}}
$$

where $X_{t}(T)$ is given by equation (18) of Theorem 1 and:

$$
\begin{aligned}
& \operatorname{Var}_{t}^{\mathbb{Q}}\left(V_{R}\right)=\frac{x_{t} \delta^{2}}{\gamma^{3} T^{2}}\left[e^{-\gamma t}\right.\left.\left(1-e^{-2 \gamma(T-t)}\right)-2(T-t) \gamma e^{-\gamma T}\right] \\
&+\frac{\delta^{2}}{2 \gamma^{3} T^{2}}\left[2 \theta_{\tau}^{2} \gamma(T-t)+\right.\left.2\left(V_{0}-\theta_{\tau}^{2}\right) \frac{\gamma}{\gamma_{\tau}} e^{-\gamma_{\tau} t}+4 \theta_{\tau}^{2} e^{-\gamma(T-t)}-\theta_{\tau}^{2} e^{-2 \gamma(T-t)}-3 \theta_{\tau}^{2}\right] \\
&-\frac{\delta^{2}\left(V_{0}-\theta_{\tau}^{2}\right)}{\gamma^{2} T^{2}\left(\gamma_{\tau}^{2}+2 \gamma^{2}-3 \gamma \gamma_{\tau}\right)}[ 2\left(\gamma_{\tau}-2 \gamma\right) e^{-\gamma(T-t)-\gamma_{\tau} t} \\
&\left.+\left(\gamma-\gamma_{\tau}\right) e^{-2 \gamma(T-t)-\gamma_{\tau} t}+2 \frac{\gamma^{2}}{\gamma_{\tau}} e^{-\gamma_{\tau} T}\right] .
\end{aligned}
$$

Proof: The (conditioned) Brockhaus\&Long approximation gives us:

$$
Y_{t}(T)=\mathbb{E}_{t}^{\mathbb{Q}}\left(\sqrt{V_{R}}\right) \approx \sqrt{\mathbb{E}_{t}^{\mathbb{Q}}\left(V_{R}\right)}-\frac{V a r_{t}^{\mathbb{Q}}\left(V_{R}\right)}{8 \mathbb{E}_{t}^{\mathbb{Q}}\left(V_{R}\right)^{\frac{3}{2}}}=\sqrt{X_{t}(T)}-\frac{V a r_{t}^{\mathbb{Q}}\left(V_{R}\right)}{8 X_{t}(T)^{\frac{3}{2}}} .
$$

Furthermore:

$$
\begin{aligned}
\operatorname{Var}_{t}^{\mathbb{Q}}\left(V_{R}\right) & =\mathbb{E}_{t}^{\mathbb{Q}}\left(\left(V_{R}-\mathbb{E}_{t}^{\mathbb{Q}}\left(V_{R}\right)\right)^{2}\right) \\
& =\frac{1}{T^{2}} \mathbb{E}_{t}^{\mathbb{Q}}\left(\left(\int_{0}^{T}\left(V_{s}-\mathbb{E}_{t}^{\mathbb{Q}}\left(V_{s}\right)\right) d s\right)^{2}\right) .
\end{aligned}
$$

From (35) we have $V_{t}=\theta_{\tau}^{2}+\left(V_{0}-\theta_{\tau}^{2}\right) e^{-\gamma_{\tau} t}+e^{-\gamma t} \tilde{W}_{\phi_{t}}$, and since $\tilde{W}_{\phi_{t}}$ is a martingale, $V_{s}-\mathbb{E}_{t}^{\mathbb{Q}}\left(V_{s}\right)=0$ if $s \leq t$, and $V_{s}-\mathbb{E}_{t}^{\mathbb{Q}}\left(V_{s}\right)=e^{-\gamma s}\left(\tilde{W}_{\phi_{s}}-x_{t}\right)$ if $s>t$.

Therefore:

$$
\begin{aligned}
& \operatorname{Var}_{t}^{\mathbb{Q}}\left(V_{R}\right)=\frac{1}{T^{2}} \mathbb{E}_{t}^{\mathbb{Q}}\left(\left(\int_{t}^{T} e^{-\gamma s}\left(\tilde{W}_{\phi_{s}}-x_{t}\right) d s\right)^{2}\right) \\
& =\frac{1}{T^{2}} x_{t}^{2}\left(\int_{t}^{T} e^{-\gamma s} d s\right)^{2}+\frac{1}{T^{2}} \mathbb{E}_{t}^{\mathbb{Q}}\left(\left(\int_{t}^{T} e^{-\gamma s} \tilde{W}_{\phi_{s}} d s\right)^{2}\right) \\
& -\frac{2}{T^{2}} x_{t}\left(\int_{t}^{T} e^{-\gamma s} \mathbb{E}_{t}^{\mathbb{Q}}\left(\tilde{W}_{\phi_{s}}\right) d s\right)\left(\int_{t}^{T} e^{-\gamma s} d s\right) .
\end{aligned}
$$

The interchange of expectation and integral in the last equation is justified the following way: by definition of $\tilde{W}_{\phi_{s}}=x_{s}$ in (21), we get:

$$
\begin{aligned}
& \mathbb{E}_{t}^{\mathbb{Q}}\left(\int_{t}^{T} e^{-\gamma s} \tilde{W}_{\phi_{s}} d s\right)=\mathbb{E}_{t}^{\mathbb{Q}}\left(\int_{t}^{T}-\left(V_{0}-\theta_{\tau}^{2}\right) e^{-\gamma_{\tau} s}+V_{s}-\theta_{\tau}^{2} d s\right) \\
= & \int_{t}^{T}-\left(V_{0}-\theta_{\tau}^{2}\right) e^{-\gamma_{\tau} s}-\theta_{\tau}^{2} d s+\mathbb{E}_{t}^{\mathbb{Q}}\left(\int_{t}^{T} V_{s} d s\right) .
\end{aligned}
$$


We can interchange expectation and integral in the latter expression by Tonelli's theorem, which gives:

$$
\begin{aligned}
& \mathbb{E}_{t}^{\mathbb{Q}}\left(\int_{t}^{T} e^{-\gamma s} \tilde{W}_{\phi_{s}} d s\right)=\int_{t}^{T}-\left(V_{0}-\theta_{\tau}^{2}\right) e^{-\gamma_{\tau} s}-\theta_{\tau}^{2} d s+\int_{t}^{T} \mathbb{E}_{t}^{\mathbb{Q}}\left(V_{s}\right) d s \\
= & \int_{t}^{T} e^{-\gamma s} \mathbb{E}_{t}^{\mathbb{Q}}\left(\tilde{W}_{\phi_{s}}\right) d s .
\end{aligned}
$$

Now we continue our computation to get:

$$
\begin{aligned}
& \operatorname{Var}_{t}^{\mathbb{Q}}\left(V_{R}\right)=-\frac{1}{T^{2}} x_{t}^{2}\left(\int_{t}^{T} e^{-\gamma s} d s\right)^{2}+\frac{1}{T^{2}} \mathbb{E}_{t}^{\mathbb{Q}}\left(\left(\int_{t}^{T} e^{-\gamma s} \tilde{W}_{\phi_{s}} d s\right)^{2}\right) \\
& =\frac{1}{T^{2}} \int_{t}^{T} \int_{t}^{T} e^{-\gamma(s+u)} \mathbb{E}_{t}^{\mathbb{Q}}\left(\tilde{W}_{\phi_{s}} \tilde{W}_{\phi_{u}}\right) d s d u-\frac{1}{T^{2}} x_{t}^{2} e^{-2 \gamma t}\left(\frac{1-e^{-\gamma(T-t)}}{\gamma}\right)^{2} .
\end{aligned}
$$

The interchange expectation-integral:

$$
\mathbb{E}_{t}^{\mathbb{Q}}\left(\int_{t}^{T} \int_{t}^{T} e^{-\gamma(s+u)} \tilde{W}_{\phi_{s}} \tilde{W}_{\phi_{u}} d s d u\right)=\int_{t}^{T} \int_{t}^{T} e^{-\gamma(s+u)} \mathbb{E}_{t}^{\mathbb{Q}}\left(\tilde{W}_{\phi_{s}} \tilde{W}_{\phi_{u}}\right) d s d u
$$

is justified the same way as above, using the definition of $\tilde{W}_{\phi_{t}}=x_{t}$ in (21) together with Tonelli's theorem. Finally, we use equation (37) of Lemma 1 and integrate the expression with respect to $s$ and $u$ to complete the proof.

Corollary 2: The Brockhaus\&Long approximation of the volatility swap price $K_{v o l}$ of maturity $T$ at initiation of the contract $t=0$ in the delayed Heston model (5)-(8) is given by:

$$
K_{v o l} \approx \sqrt{K_{v a r}}-\frac{\operatorname{Var}_{0}^{\mathbb{Q}}\left(V_{R}\right)}{8 K_{v a r}^{\frac{3}{2}}}
$$

where $K_{v a r}$ is given by formula (20) of Corollary 1 and:

$$
\begin{gathered}
\operatorname{Var}_{0}^{\mathbb{Q}}\left(V_{R}\right)=\frac{\delta^{2} e^{-2 \gamma T}}{2 T^{2} \gamma^{3}}\left[\theta_{\tau}^{2}\left(2 \gamma T e^{2 \gamma T}+4 e^{\gamma T}-3 e^{2 \gamma T}-1\right)+\frac{\gamma}{2 \gamma-\gamma_{\tau}}\left(V_{0}-\theta_{\tau}^{2}\right)\right. \\
\left.\left(2 e^{2 \gamma T}\left(2 \frac{\gamma}{\gamma_{\tau}}-1\right)-4 \gamma e^{\gamma T}\left(\frac{e^{\left(\gamma-\gamma_{\tau}\right) T}-1}{\gamma-\gamma_{\tau}}\right)+4 e^{\gamma T}\left(1-\frac{\gamma}{\gamma_{\tau}} e^{\left(\gamma-\gamma_{\tau}\right) T}\right)-2\right)\right] .
\end{gathered}
$$

We notice that letting $\tau \rightarrow 0$ (and therefore $\gamma_{\tau} \rightarrow \gamma$ ) we get the formula of Swishchuk [22].

Proof: We have by definition $K_{v o l}=Y_{0}(T)$, therefore the result is obtained from equation (40) of Theorem 2. 


\section{$5 \quad$ Volatility Swap Hedging}

In this section, we consider dynamic hedging of volatility swaps using variance swaps, as the latter are a fairly liquid, easy to trade derivatives. In the spirit of Broadie and Jain [6], we consider a portfolio containing at time $t$ one unit of volatility swap and $\beta_{t}$ units of variance swaps, both of maturity $T$. Therefore the value $\Pi_{t}$ of the portfolio at time $t$ is:

$$
\Pi_{t}=e^{-r(T-t)}\left[Y_{t}(T)-K_{v o l}+\beta_{t}\left(X_{t}(T)-K_{v a r}\right)\right] .
$$

The portfolio is self-financing, therefore:

$$
d \Pi_{t}=r \Pi_{t} d t+e^{-r(T-t)}\left[d Y_{t}(T)+\beta_{t} d X_{t}(T)\right] .
$$

The price processes $X_{t}(T)$ and $Y_{t}(T)$ can be expressed, denoting $I_{t}:=\int_{0}^{t} V_{s} d s$ the accumulated variance at time $t$ (known at this time):

$$
\begin{aligned}
& X_{t}(T)=\mathbb{E}_{t}^{\mathbb{Q}}\left[\frac{1}{T} I_{t}+\frac{1}{T} \int_{t}^{T} V_{s} d s\right]=g\left(t, I_{t}, V_{t}\right), \\
& Y_{t}(T)=\mathbb{E}_{t}^{\mathbb{Q}}\left[\sqrt{\left.\frac{1}{T} I_{t}+\frac{1}{T} \int_{t}^{T} V_{s} d s\right]=h\left(t, I_{t}, V_{t}\right) .}\right.
\end{aligned}
$$

Remembering that $\tilde{\theta}_{t}^{2}=\theta_{\tau}^{2}+\left(V_{0}-\theta_{\tau}^{2}\right) \frac{\left(\gamma-\gamma_{\tau}\right)}{\gamma} e^{-\gamma_{\tau} t}$ and noticing that $d I_{t}=V_{t} d t$, by Ito's lemma we get:

$$
\begin{aligned}
& d X_{t}(T)=\left[\frac{\partial g}{\partial t}+\frac{\partial g}{\partial I_{t}} V_{t}+\frac{\partial g}{\partial V_{t}} \gamma\left(\tilde{\theta}_{t}^{2}-V_{t}\right)+\frac{1}{2} \frac{\partial^{2} g}{\partial V_{t}^{2}} \delta^{2} V_{t}\right] d t+\frac{\partial g}{\partial V_{t}} \delta \sqrt{V_{t}} d W_{t}^{\mathbb{Q}}, \\
& d Y_{t}(T)=\left[\frac{\partial h}{\partial t}+\frac{\partial h}{\partial I_{t}} V_{t}+\frac{\partial h}{\partial V_{t}} \gamma\left(\tilde{\theta}_{t}^{2}-V_{t}\right)+\frac{1}{2} \frac{\partial^{2} h}{\partial V_{t}^{2}} \delta^{2} V_{t}\right] d t+\frac{\partial h}{\partial V_{t}} \delta \sqrt{V_{t}} d W_{t}^{\mathbb{Q}} .
\end{aligned}
$$

As conditional expectations of cashflows at maturity of the contract, the price processes $X_{t}(T)$ and $Y_{t}(T)$ are by construction martingales, and therefore we should have:

$$
\begin{aligned}
& \frac{\partial g}{\partial t}+\frac{\partial g}{\partial I_{t}} V_{t}+\frac{\partial g}{\partial V_{t}} \gamma\left(\tilde{\theta}_{t}^{2}-V_{t}\right)+\frac{1}{2} \frac{\partial^{2} g}{\partial V_{t}^{2}} \delta^{2} V_{t}=0, \\
& \frac{\partial h}{\partial t}+\frac{\partial h}{\partial I_{t}} V_{t}+\frac{\partial h}{\partial V_{t}} \gamma\left(\tilde{\theta}_{t}^{2}-V_{t}\right)+\frac{1}{2} \frac{\partial^{2} h}{\partial V_{t}^{2}} \delta^{2} V_{t}=0 .
\end{aligned}
$$

The second equation, combined with some appropriate boundary conditions, was used in Broadie and Jain [6] to compute the value of the price process $Y_{t}(T)$, whereas we focus on its Brockhaus\&Long approximation. 
Therefore we get:

$$
\begin{aligned}
& d X_{t}(T)=\frac{\partial g}{\partial V_{t}} \delta \sqrt{V_{t}} d W_{t}^{\mathbb{Q}}, \\
& d Y_{t}(T)=\frac{\partial h}{\partial V_{t}} \delta \sqrt{V_{t}} d W_{t}^{\mathbb{Q}} .
\end{aligned}
$$

and so:

$$
d \Pi_{t}=r \Pi_{t} d t+e^{-r(T-t)}\left[\frac{\partial h}{\partial V_{t}} \delta \sqrt{V_{t}} d W_{t}^{\mathbb{Q}}+\beta_{t} \frac{\partial g}{\partial V_{t}} \delta \sqrt{V_{t}} d W_{t}^{\mathbb{Q}}\right] .
$$

In order to dynamically hedge a volatility swap of maturity $T$, one should therefore hold $\beta_{t}$ units of variance swap of maturity $T$, with:

$$
\beta_{t}=-\frac{\frac{\partial h}{\partial V_{t}}}{\frac{\partial g}{\partial V_{t}}}=-\frac{\frac{\partial Y_{t}(T)}{\partial V_{t}}}{\frac{\partial X_{t}(T)}{\partial V_{t}}} .
$$

Remembering that $\operatorname{Var}_{0}^{\mathbb{Q}}\left(V_{R}\right), K_{\text {var }}$ are given respectively in Corollary 2 and 1 , the initial hedge ratio $\beta_{0}$ is given by:

$$
\begin{aligned}
& \beta_{0}=-\frac{\frac{\partial Y_{0}(T)}{\partial V_{0}}}{\frac{\partial X_{0}(T)}{\partial V_{0}}}, \\
& \frac{\partial X_{0}(T)}{\partial V_{0}}=\frac{1-e^{-\gamma_{\tau} T}}{\gamma_{\tau} T}, \\
& \frac{\partial Y_{0}(T)}{\partial V_{0}} \approx \frac{\frac{\partial X_{0}(T)}{\partial V_{0}}}{2 \sqrt{K_{v a r}}}-\frac{K_{\text {var }} \frac{\partial \operatorname{Var} r_{0}^{\mathbb{Q}}\left(V_{R}\right)}{\partial V_{0}}-\frac{3}{2} \frac{\partial X_{0}(T)}{\partial V_{0}} \operatorname{Var}_{0}^{\mathbb{Q}}\left(V_{R}\right)}{8 K_{v a r}^{\frac{5}{2}}}, \\
& \frac{\partial \operatorname{Var}_{0}^{\mathbb{Q}}\left(V_{R}\right)}{\partial V_{0}}=\frac{\delta^{2} e^{-2 \gamma T}}{T^{2} \gamma^{3}} \frac{\gamma}{2 \gamma-\gamma_{\tau}}\left[e^{2 \gamma T}\left(2 \frac{\gamma}{\gamma_{\tau}}-1\right)\right. \\
& \left.-2 \gamma e^{\gamma T}\left(\frac{e^{\left(\gamma-\gamma_{\tau}\right) T}-1}{\gamma-\gamma_{\tau}}\right)+2 e^{\gamma T}\left(1-\frac{\gamma}{\gamma_{\tau}} e^{\left(\gamma-\gamma_{\tau}\right) T}\right)-1\right] .
\end{aligned}
$$

Remembering that $\operatorname{Var}_{t}^{\mathbb{Q}}\left(V_{R}\right), X_{t}(T)$ are given respectively in Theorems 2 and 1 , the hedge ratio $\beta_{t}$ for $t>0$ is given by:

$$
\begin{aligned}
& \beta_{t}=-\frac{\frac{\partial Y_{t}(T)}{\partial V_{t}}}{\frac{\partial X_{t}(T)}{\partial V_{t}}}, \\
& \frac{\partial X_{t}(T)}{\partial V_{t}}=\frac{1-e^{-\gamma(T-t)}}{\gamma T}, \\
& \frac{\partial Y_{t}(T)}{\partial V_{t}} \approx \frac{\frac{\partial X_{t}(T)}{\partial V_{t}}}{2 \sqrt{X_{t}(T)}}-\frac{X_{t}(T) \frac{\partial V a r_{t}^{\mathbb{Q}}\left(V_{R}\right)}{\partial V_{t}}-\frac{3}{2} \frac{\partial X_{t}(T)}{\partial V_{t}} \operatorname{Var}_{t}^{\mathbb{Q}}\left(V_{R}\right)}{8 X_{t}(T)^{\frac{5}{2}}}, \\
& \frac{\partial V a r_{t}^{\mathbb{Q}}\left(V_{R}\right)}{\partial V_{t}}=\frac{\delta^{2}}{\gamma^{3} T^{2}}\left[1-e^{-2 \gamma(T-t)}-2(T-t) \gamma e^{-\gamma(T-t)}\right] .
\end{aligned}
$$


We take the parameters that have been calibrated in section 3 on September $30^{\text {th }} 2011$ and we plot the naive Volatility Swap strike $\sqrt{K_{\text {var }}}$ together with the adjusted Volatility Swap strike $\sqrt{K_{\text {var }}}-\frac{V a r^{\mathbb{Q}}\left(V_{R}\right)}{8 K_{\text {var }}^{\frac{3}{2}}}$ along the maturity dimension, as well as the convexity adjustment $\frac{\operatorname{Var}^{\mathbb{Q}}\left(V_{R}\right)}{8 K_{v a r}^{\frac{3}{2}}}$ :

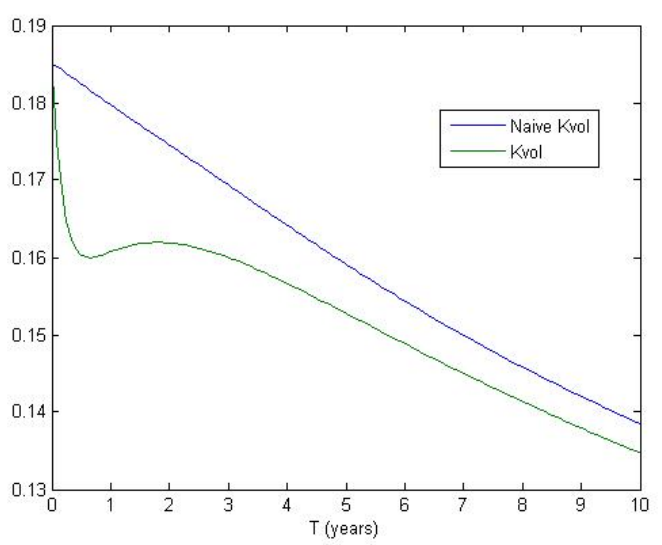

Figure 1: Naive Volatility Swap Strike Vs. Adjusted Volatility Swap Strike

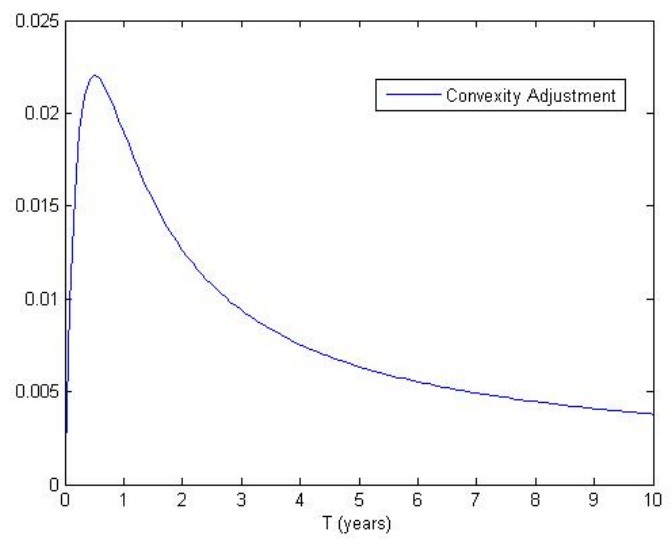

Figure 2: Convexity Adjustment

The naive Volatility Swap Strike represents the initial fair value of the volatility swap contract obtained without taking into account the convexity adjustment $\frac{\operatorname{Var}^{\mathbb{Q}}\left(V_{R}\right)}{8 K_{v a r}^{\frac{3}{2}}}$ linked to the Brockhaus\&Long approximation, whereas the adjusted Volatility Swap Strike represents this initial fair value when we do take into account the convexity adjustment. The difference between the former and the latter is quantified by the convexity adjustment and is represented on the second graphic. We see that neglecting the convexity adjustment leads to an overpricing of the volatility swap. On this example, the overpricing is especially significant for maturities less than $2 \mathrm{Y}$, with a peak difference of more than $2 \%$ between the naive and adjusted strikes for maturities around $6 \mathrm{M}$. The position of this local extremum (here, around $6 \mathrm{M}$ ) is linked to the values of the 
calibrated parameters and therefore varies depending on the date we perform the calibration at.

We also plot the initial hedge ratio $\beta_{0}$ along the maturity dimension:

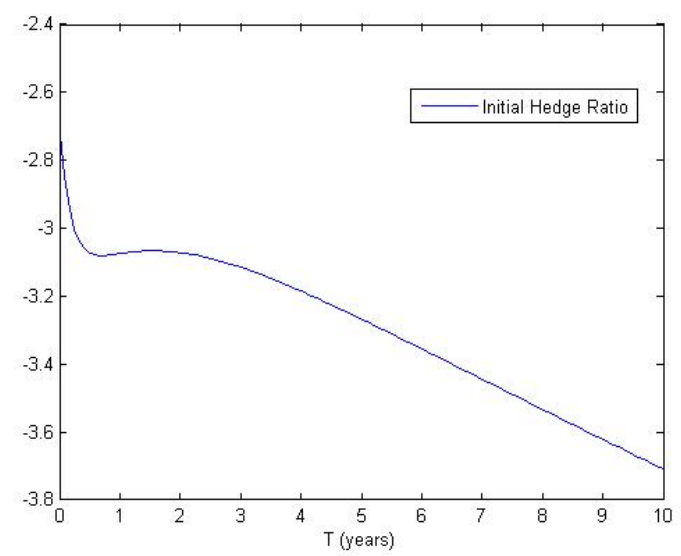

Figure 3: Initial Hedge Ratio

This initial hedge ratio $\beta_{0}$ represents the quantity of variance swap contracts we need to buy (if $\beta_{0}>0$ ) or sell (if $\beta_{0}<0$ ) to hedge our position on one volatility swap contract of the same maturity. Of course, in order to cancel the risk, $\beta_{0}$ has to be negative if we buy a volatility swap contract, and positive if we sell one. Here we have assumed that we hold a long position on a volatility swap contract, i.e. that we have bought one such contract. The plot tells us that for one volatility swap contract bought, we need to sell approximately 3 variance swap contracts of the same maturity (depending of the maturity of the contract) to hedge our position on the volatility swap, i.e. to cancel the risk inherent to our position. We say that we hold a short position on the variance swap contracts. The trend is that the higher the maturity of the volatility swap contract, the more variance swap contracts we need to sell in order to hedge our position. This was to be expected because for such pure volatility contracts, the longer the maturity, the higher the probability that the volatility varies significantly, i.e. the higher the risk.

\section{Conclusions}

In this paper, we introduced a variance drift adjusted version of the Heston model based on the concept of delay, the Delayed Heston model (section 2). As explained in the introduction, this model makes a bridge between the popular Heston model and the delayed stochastic volatility model considered by Swishchuk in [23]. Our model has two additional parameters compared to the Heston model and since it can be seen as a time-dependent Heston model with time-dependent long-range variance $\tilde{\theta}_{t}^{2}$, it can be implemented very easily, for 
both Monte Carlo simulation and pricing of call options via the semi-closed formulas which can be derived (see Appendix A). We calibrated our model on 12 dates ranging from Sep. $19^{\text {th }}$ to Oct. $17^{\text {th }} 2011$ for the FOREX underlying EURUSD (section 3). Our findings were twofold: the Delayed Heston model always outperformed significantly the Heston model in terms of average (absolute) calibration error (especially for long maturities and ATM options), but also in terms of the standard deviation of the calibration errors. The latter is highly desirable in practice as we do not want to face the case of very poorly priced options on one hand, and almost perfectly priced options on the other: it is better that each individual calibration error corresponding to each call option is close to the average calibration error, and therefore that the standard deviation of the calibration errors is low. In sections 4 and 5, we considered respectively the pricing of variance and volatility swaps, and the dynamic hedging of a position on a volatility swap by a position on variance swaps, the latter being very liquid financial derivatives. We obtained a closed formula for both the price process of the variance swap and the Brockhaus\&Long approximation (which is a $2^{\text {nd }}$ order approximation) of the price process of the volatility swap. Finally, to illustrate these last sections, we displayed 3 graphics showing the importance of taking into account the convexity adjustment (corresponding to the Brockhaus\&Long approximation) when pricing a volatility swap, and that taking naively the volatility swap strike $K_{v o l}$ to be $\sqrt{K_{v a r}}$ may lead to a significant mispricing of the volatility swap. Regarding future research directions, it would be interesting to understand how the Delayed Heston model could be improved by considering a Stochastic Local Volatility (SLV) approach, or how we could improve calibration on call option prices using the Generalized Methods of Moments (GMM), for example. Another interesting direction would be to use our model for forecasting purposes (instead of pricing), and estimate our model parameters via the computation of the Delayed Heston volatility likelihood function, which would have to be derived.

\section{A Semi-closed formulas for call options in the Delayed Heston Model}

From Kahl\&Jäckel [13], we get equations (71) to (74) for the price of a call option with maturity $T$ and strike $K$ in the time-dependent long-range variance Heston model:

$$
\begin{aligned}
& C_{0}=e^{-r T}\left[\frac{1}{2}(F-K)+\frac{1}{\pi} \int_{0}^{\infty}\left(F h_{1}(u)-K h_{2}(u)\right) d u\right], \\
& h_{1}(u)=\Re\left(\frac{e^{-i u \ln (K)} \varphi(u-i)}{i u F}\right), \\
& h_{2}(u)=\Re\left(\frac{e^{-i u \ln (K)} \varphi(u)}{i u}\right),
\end{aligned}
$$

with $F=S_{0} e^{(r-q) T}$ and: 


$$
\varphi(u)=e^{C(T, u)+V_{0} D(T, u)+i u \ln (F)} .
$$

By Michailov\&Nöegel [20], we have that $C(t, u)$ and $D(t, u)$ solve the following differential equations:

$$
\begin{aligned}
& \frac{d C(t, u)}{d t}=\gamma \tilde{\theta}_{t}^{2} D(t, u) \\
& \frac{d D(t, u)}{d t}-\frac{\delta^{2}}{2} D^{2}(t, u)+(\gamma-i u \rho \delta) D(t, u)+\frac{1}{2}\left(u^{2}+i u\right)=0 \\
& C(0, u)=D(0, u)=0 .
\end{aligned}
$$

The Riccati equation for $D(t, u)$ doesn't depend on $\tilde{\theta}_{t}^{2}$, therefore its solution is just the solution of the classical Heston model given in Kahl\&Jäckel [13]:

$$
\begin{aligned}
& D(t, u)=\frac{\gamma-i \rho \delta u+d}{\delta^{2}}\left[\frac{1-e^{d t}}{1-g e^{d t}}\right] \\
& g=\frac{\gamma-i \rho \delta u+d}{\gamma-i \rho \delta u-d} \\
& d=\sqrt{(\gamma-i \rho \delta u)^{2}+\delta^{2}\left(i u+u^{2}\right)} .
\end{aligned}
$$

Given $D(t, u)$ and the definition of $\tilde{\theta}_{t}^{2}$, we can compute $C(t, u)$ from $(75)$ and $(77)$ :

$$
C(t, u)=\gamma \theta_{\tau}^{2} f(t, u)+\left(V_{0}-\theta_{\tau}^{2}\right)\left(\gamma-\gamma_{\tau}\right) \int_{0}^{t} e^{-\gamma_{\tau} s} D(s, u) d s
$$

Where $f(t, u)=\int_{0}^{t} D(s, u) d s$ is given in Kahl\&Jäckel [13]:

$$
f(t, u)=\frac{1}{\delta^{2}}\left((\gamma-i \rho \delta u+d) t-2 \ln \left(\frac{1-g e^{d t}}{1-g}\right)\right) .
$$

Unfortunately, the integral $\int_{0}^{t} e^{-\gamma_{\tau} s} D(s, u) d s$ in (81) cannot be computed directly as $\int_{0}^{t} D(s, u) d s$. The logarithm in $f(t, u)$ can be handled as suggested in Kahl\&Jäckel [13], as well as the integration of the Heston integral, namely: 


$$
\begin{aligned}
& C_{0}=e^{-r T} \int_{0}^{1} y(x) d x, \\
& y(x)=\frac{1}{2}(F-K)+\frac{F h_{1}\left(-\frac{\ln (x)}{C_{\infty}}\right)-K h_{2}\left(-\frac{\ln (x)}{C_{\infty}}\right)}{x \pi C_{\infty}},
\end{aligned}
$$

where $C_{\infty}>0$ is an integration constant.

The following limit conditions are given in Kahl\&Jäckel [13]:

$$
\begin{aligned}
& \lim _{x \rightarrow 0} y(x)=\frac{1}{2}(F-K), \\
& \lim _{x \rightarrow 1} y(x)=\frac{1}{2}(F-K)+\frac{F H_{1}-K H_{2}}{\pi C_{\infty}}, \\
& H_{j}=\lim _{u \rightarrow 0} h_{j}(u)=\ln \left(\frac{F}{K}\right)+\tilde{c}_{j}(T)+V_{0} \tilde{d}_{j}(T),
\end{aligned}
$$

where:

$$
\begin{aligned}
& \tilde{d}_{1}(t)=\Im\left(\frac{\partial D}{\partial u}(t,-i)\right), \\
& \tilde{c}_{1}(t)=\Im\left(\frac{\partial C}{\partial u}(t,-i)\right), \\
& \tilde{d}_{2}(t)=\Im\left(\frac{\partial D}{\partial u}(t, 0)\right), \\
& \tilde{c}_{2}(t)=\Im\left(\frac{\partial C}{\partial u}(t, 0)\right) .
\end{aligned}
$$

Expressions for $\tilde{d}_{1}(t)$ and $\tilde{d}_{2}(t)$ are the same as in Kahl\&Jäckel [13] as $\tilde{\theta}_{t}^{2}$ doesn't play any role in them. Given (75) and (77), we compute $\tilde{c}_{1}(T)$ and $\tilde{c}_{2}(T)$ in our time-dependent long-range variance Heston model by:

$$
\tilde{c}_{j}(T)=\gamma \int_{0}^{T} \tilde{\theta}_{t}^{2} \tilde{d}_{j}(t) d t
$$

After computing the integrals we get:

If $\gamma-\rho \delta \neq 0$ and $\gamma-\rho \delta+\gamma_{\tau} \neq 0$ :

$$
\begin{aligned}
& \tilde{d}_{1}(T)=\frac{1-e^{-(\gamma-\rho \delta) T}}{2(\gamma-\rho \delta)} \\
& \tilde{c}_{1}(T)=\gamma \theta_{\tau}^{2} \frac{e^{-(\gamma-\rho \delta) T}-1+(\gamma-\rho \delta) T}{2(\gamma-\rho \delta)^{2}} \\
& +\frac{\left(V_{0}-\theta_{\tau}^{2}\right)\left(\gamma-\gamma_{\tau}\right)}{2(\gamma-\rho \delta)}\left(-\frac{e^{-\gamma_{\tau} T}-1}{\gamma_{\tau}}+\frac{e^{-\left(\gamma-\rho \delta+\gamma_{\tau}\right) T}-1}{\gamma-\rho \delta+\gamma_{\tau}}\right) .
\end{aligned}
$$


If $\gamma-\rho \delta \neq 0$ and $\gamma-\rho \delta+\gamma_{\tau}=0$ :

$$
\begin{aligned}
& \tilde{d}_{1}(T)=\frac{1-e^{-(\gamma-\rho \delta) T}}{2(\gamma-\rho \delta)} \\
& \tilde{c}_{1}(T)=\gamma \theta_{\tau}^{2} \frac{e^{-(\gamma-\rho \delta) T}-1+(\gamma-\rho \delta) T}{2(\gamma-\rho \delta)^{2}} \\
& +\frac{\left(V_{0}-\theta_{\tau}^{2}\right)\left(\gamma-\gamma_{\tau}\right)}{2(\gamma-\rho \delta)}\left(-\frac{e^{-\gamma_{\tau} T}-1}{\gamma_{\tau}}-T\right) .
\end{aligned}
$$

If $\gamma-\rho \delta=0$ :

$$
\begin{aligned}
& \tilde{d}_{1}(T)=\frac{T}{2}, \\
& \tilde{c}_{1}(T)=\gamma \theta_{\tau}^{2} \frac{T^{2}}{4}+\frac{\left(V_{0}-\theta_{\tau}^{2}\right)\left(\gamma-\gamma_{\tau}\right)}{2}\left(-\frac{T e^{-\gamma_{\tau} T}}{\gamma_{\tau}}+\frac{1-e^{-\gamma_{\tau} T}}{\gamma_{\tau}^{2}}\right),
\end{aligned}
$$

and:

$$
\begin{aligned}
& \tilde{d}_{2}(T)=\frac{e^{-\gamma T}-1}{2 \gamma} \\
& \tilde{c}_{2}(T)=\gamma \theta_{\tau}^{2} \frac{1-e^{-\gamma T}-\gamma T}{2 \gamma^{2}} \\
& +\frac{\left(V_{0}-\theta_{\tau}^{2}\right)\left(\gamma-\gamma_{\tau}\right)}{2 \gamma}\left(-\frac{1-e^{-\gamma_{\tau} T}}{\gamma_{\tau}}-\frac{e^{\left(-\gamma_{\tau}-\gamma\right) T}-1}{\gamma_{\tau}+\gamma}\right) .
\end{aligned}
$$

\section{References}

1.Arriojas, M., Hu, Y., Mohammed, S-E., Pap, G. A Delayed Black and Scholes Formula Stochastic Analysis and Applications 25:2, 471-492, 2007.

2.Bell, D. R., Mohammed, S-E. The Malliavin calculus and stochastic delay equations. J. Funct. Anal. 99, no. 1, 75-99, 1991.

3.Bell, D. R., Mohammed, S-E. Smooth densities for degenerate stochastic delay equations with hereditary drift. Ann. Probab. 23, no. 4, 1875-1894, 1995.

4.Bollerslev, T. Generalized autoregressive conditional heteroscedasticity. J. Economics 31, 307-27, 1986.

5.Broadie, M. and Jain, A. The effect of jumps and discrete sampling on volatility and variance swaps. Intern. J. Theor. Appl. Finance, vol. 11, no. 8, pp. 761-797, 2008.

6.Broadie, M and Jain, A. Pricing and Hedging Volatility Derivatives. Journal of Derivatives, Vol.15, No.3, 7-24, 2008.

7.Brockhaus, O. and Long, D. Volatility swaps made simple. Risk, January, 92-96, 2000. 
8.Carr, P. and Lee, R. Volatility Derivatives. Annu. Rev, Financ. Econ., 1:1-21 (doi:10.1146/annurev.financial.050808.114304), 2009.

9.Filipovic, D. Affine Variance Swap Curve Models. Seminar on Stochastic Analysis, Random Fields and Applications VII, Progress in Probability, 67, 381-393, Springer Basel, 2013.

10.Hao, J. and Zhang, J.E. GARCH option pricing models, the CBOE VIX, and Variance Risk premium. Journal of Financial Econometrics, 11(3):556-580, 2013.

11.Heston, S. A closed-form solution for options with stochastic volatility with applications to bond and currency options. Review of Financial Studies, 6, 327-343, 1993.

12.Heston, S. and Nandi, S. Derivatives on volatility: some simple solutions based on observables. Federal Reserve Bank of Atlanta, working paper 2000-20, November, 2000 .

13.Kahl, C. and Jäckel, P. Not-so-complex logarithms in the Heston model. Wilmott Magazine, September, pp. 94-103, 2005.

14.Karatzas, I. and Shreve, S.E., Brownian motion and Stochastic Calculus, $2^{\text {nd }}$ Edition, Springer Science+Business Media, LLC, 1998.

15.Kazmerchuk, Y., Swishchuk, A. and Wu, J. A continuous-time GARCH model for stochastic volatility with delay. Candian Appl. Math. Quart., v. 13, No. 2, 2005.

16.Kazmerchuk, Y., Swishchuk, A. and Wu, J. The pricing of options for security markets with delayed response. Mathematics and Computers in Simulation 75 6979, 2007.

17.Kind, P., Lipster, R. S., Runggaldier, W. J.. Diffusion approximation in past dependent models and applications to option pricing. The Annals of Applied Probability vol.1, no. 3, pp. 379-405, 1991.

18.Kruse, S. and Nögel, U. On the pricing of forward starting options in Heston's model on stochastic volatility. Finance Stochast. 9, 233250, 2005.

19.Mikhailov, S. and Noegel, U. Heston's Stochastic Volatility Model Implementation, Calibration and Some Extensions. Wilmott Magazine, 1, pp. 74-79, 2005.

20.Mikhailov, S. and Noegel, U. Heston's Stochastic Volatility Model Implementation, Calibration and Some Extensions. Wilmott Magazine, 1, pp. 74-79, 2005.

21.Swishchuk, A. and Li, X. Pricing variance swaps for stochastic volatilities with delay and jumps, Intern. J. Stoch. Anal., vol. 2011 (2011), Article ID 435145, 2011.

22.Swishchuk, A. Modeling of variance and volatility swaps for financial markets with Stochastic volatilities. Wilmott Magazine, September issue, No. 2, pp. 64-72, 2004.

23.Swishchuk, A. Modeling and pricing of variance swaps for stochastic volatilities with delay. Wilmott Magazine, September, No. 19, pp. 63-73, 2005.

24.Swishchuk, A. Modeling and Pricing of Variance Swaps for Multi-Factor Stochastic Volatilities with Delay. Canad. Appl. Math. Quart., Volume 14, Number 4, Winter 2006.

25.Swishchuk, A. Variance and volatility swaps in energy markets. J. Energy Markets, Volume 6/Number 1, Spring 2013 (3349), 2013.

26.Swishchuk, A and Malenfant, K. Variance swap for local Levy based stochastic volatility with delay. Intern. Rev. Appl. Fin. Iss. Econ. (IRAFIE), Vol. 3, No. 2, 2011, pp. 432-441, 2011.

27.Zhang, J.E. and Zhu, Y. Vix Futures. The Journal of Futures Markets, 2(6):521$531,2006$. 